PROCEEDINGS OF THE

AMERICAN MATHEMATICAL SOCIETY

Volume 130, Number 1, Pages 163-166

S 0002-9939(01)06039-7

Article electronically published on June 8, 2001

\title{
THE HOPF CONJECTURE FOR MANIFOLDS WITH LOW COHOMOGENEITY OR HIGH SYMMETRY RANK
}

\author{
THOMAS PÜTTMANN AND CATHERINE SEARLE
}

(Communicated by Wolfgang Ziller)

\begin{abstract}
We prove that the Euler characteristic of an even-dimensional compact manifold with positive (nonnegative) sectional curvature is positive (nonnegative) provided that the manifold admits an isometric action of a compact Lie group $G$ with principal isotropy group $H$ and cohomogeneity $k$ such that $k-(\operatorname{rank} G-\operatorname{rank} H) \leq 5$. Moreover, we prove that the Euler characteristic of a compact Riemannian manifold $M^{4 l+4}$ or $M^{4 l+2}$ with positive sectional curvature is positive if $M$ admits an effective isometric action of a torus $T^{l}$, i.e., if the symmetry rank of $M$ is $\geq l$.
\end{abstract}

The Gauss-Bonnet theorem states that the Euler characteristic of a closed surface $M$ is determined by its total curvature:

$$
\chi(M)=2 \pi \int_{M} K
$$

In particular, if the curvature is positive (nonnegative), the Euler characteristic of the surface is positive (nonnegative). H. Hopf [H] generalized in 1925 the GaussBonnet theorem to even-dimensional hypersurfaces of Euclidean space and posed in the early 1930's (according to Berger [Be]) the question of whether a compact even-dimensional manifold which admits a metric of positive (nonnegative) sectional curvature must have positive (nonnegative) Euler characteristic.

Indications that the Hopf conjecture should be true came from the generalizations of the Gauss-Bonnet theorem: Fenchel $[\mathrm{F}$ and Allendoerfer $[\mathrm{A}$ proved in 1940 independently a Gauss-Bonnet formula for submanifolds of Euclidean space with arbitrary codimension. Three years later Allendoerfer and Weil [AW] (using E. Cartan's result that any Riemannian manifold can locally be embedded into Euclidean space) established the theorem in its final intrinsic version: For any even-dimensional manifold the Euler characteristic can be obtained by integrating a function derived from the curvature tensor, the so-called Gauss-Bonnet integrand. Chern [C1] gave the first intrinsic proof of this theorem in 1944.

After this, many attempts were made to settle the stronger algebraic Hopf conjecture: A curvature tensor with positive (nonnegative) sectional curvature yields

Received by the editors May 16, 2000.

2000 Mathematics Subject Classification. Primary 53C20.

This paper has its roots in the nonnegative curvature seminar at the University of Pennsylvania in the academic year 1999-2000. The first named author would like to thank the University of Pennsylvania for their hospitality and A. Rigas, B. Wilking, and W. Ziller for advice and many interesting discussions during this time. The second named author was supported in part by a grant from CONACYT project number 28491-E.

(C)2001 American Mathematical Society 
a positive (nonnegative) Gauss-Bonnet integrand. Milnor (unpublished, see [C2]) actually proved the algebraic Hopf conjecture in dimension 4, but finally in 1976 Geroch [G] found curvature tensors with positive sectional curvature in all even dimensions $\geq 6$ that do not provide a positive Gauss-Bonnet integrand.

A different approach to the Hopf conjecture is to consider first Riemannian manifolds that have a certain amount of symmetry. Hopf himself and Samelson [HS] proved in 1941 that the Euler characteristic of every compact homogeneous space $G / H$ is nonnegative and positive if and only if $\operatorname{rank} G=\operatorname{rank} H$ holds. The key observations in their proof are that a regular element in the compact Lie group $G$ has at most finitely many fixed points in the homogeneous space $G / H$ and that each of these fixed points has fixed point index 1. In 1972 Wallach [W] then showed that for any even-dimensional homogeneous space of positive sectional curvature one actually has $\operatorname{rank} G=\operatorname{rank} H$. Therefore, the Hopf conjecture is true for homogeneous spaces. Recently, Podestà and Verdiani [PV] proved among other things that the Hopf conjecture also holds for cohomogeneity one manifolds. We show that much weaker symmetry assumptions are sufficient.

Theorem 1. Let $M$ be a compact even-dimensional Riemannian manifold with positive (nonnegative) sectional curvature. Let $G \times M \rightarrow M$ be an isometric action of a compact Lie group $G$ with principal isotropy group $H$ and cohomogeneity $k$. If

$$
k-(\operatorname{rank} G-\operatorname{rank} H) \leq 5,
$$

then $M$ has positive (nonnegative) Euler characteristic.

Proof. If $M$ is nonorientable, then the action of $G$ can be lifted to an action by orientation preserving isometries (see [ $\mathrm{Br}$, Corollary I.9.4]) on the orientable double covering space of $M$. We can therefore assume that $M$ is orientable.

We consider the fixed point set

$$
M^{T}=\{p \in M \mid \psi(p)=p \text { for all } \psi \in T\}
$$

of a maximal torus $T$ of $G$. Note that $M^{T}$ is equal to the fixed point set of a generating element $\psi \in T$, i.e., of an element $\psi$ with $\overline{\left\{\psi^{m} \mid m \in \mathbb{Z}\right\}}=T$. If the fixed point set $M^{T}$ is empty, then there exists a Killing field without zeros. This implies that $M$ cannot have positive sectional curvature (by Berger's theorem, see e.g. [W] ) and that the Euler characteristic is zero. We can therefore assume that $M^{T}$ is nonempty. Now each of the finitely many components of $M^{T}$ is a totally geodesic submanifold of $M$ with even codimension and the Euler characteristic of $M$ is the sum of the Euler characteristics of the components (see [K] Chapter II]). By Theorem IV.5.3 of $[\mathrm{Br}]$ each component $N$ of $M^{T}$ satisfies

$$
\operatorname{dim} N \leq k-(\operatorname{rank} G-\operatorname{rank} H) \leq 5 .
$$

Since $N$ is even-dimensional and the Hopf conjecture holds in dimensions 2 and 4 we are done.

Note that $k-(\operatorname{rank} G-\operatorname{rank} H) \leq \operatorname{dim} M-2 \operatorname{rank} G(\operatorname{see}[\mathrm{Br}$, Corollary IV.5.4]) if the action of $G$ is effective. Hence we get as a special case of Theorem 1 that any compact even-dimensional Riemannian manifold $M^{2 l+4}$ with positive (nonnegative) sectional curvature has positive (nonnegative) Euler characteristic if $M^{2 l+4}$ admits an effective isometric torus action $T^{l} \times M \rightarrow M$. Using a result from [GS] we can improve this result in the case of positive sectional curvature. 
Theorem 2. Let $M^{4 l+2}$ or $M^{4 l+4}$ be a Riemannian manifold with positive sectional curvature that admits an almost effective isometric $T^{l}$-action. Then for any $T^{1} \subset T^{l}$ the Euler characteristics of all the components of the fixed point set $\operatorname{Fix}\left(M ; T^{1}\right)$ are positive. In particular, $\chi(M)>0$.

Proof. As above we can assume that $M$ is orientable in order to have even-dimensional fixed point sets. The proof is done by induction. For $l=0$ note that the Hopf conjecture is true in dimensions 2 and 4 . For the induction step consider $M^{4 l+6}$ or $M^{4 l+8}$ with an almost effective $T^{l+1}$-action. Consider any circle $T^{1} \subset T^{l+1}$ and any component $N$ of its fixed point set in $M$. We will show that $\chi(N)>0$. Choose a $\tilde{T}^{1} \subset T^{l+1}$ such that $N \subset \operatorname{Fix}\left(M ; \tilde{T}^{1}\right)$ and such that the component $\tilde{N}$ of $\operatorname{Fix}\left(M ; \tilde{T}^{1}\right)$ that contains $N$ has maximal dimension. It follows from the slice theorem and from the representation theory of tori that $T^{l}=T^{l+1} / \tilde{T}^{1}$ acts almost effectively on $\tilde{N}$. If $\operatorname{codim} \tilde{N} \geq 4$, then we know from the induction assumption that in particular $N$ as a component of $\operatorname{Fix}\left(\tilde{N} ; T^{1}\right)$ has positive Euler characteristic and hence we are done. In the case where $\operatorname{codim} \tilde{N}=2$ we know from [GS] that $M$ is differentiably covered by a sphere or a complex projective space. From results of Bredon [Br. Chapters III and VII] it follows that all the components of the fixed point set of any circle action on $M$ have positive Euler characteristic. Thus in particular $N$ has positive Euler characteristic.

After this paper was accepted for publication we were informed that Xiaochun Rong obtained Theorem[2independently (see $[\underline{R}]$ ). In his paper he gives many more results on the topology of positively curved manifolds with high symmetry rank.

\section{REFERENCES}

[A] C. B. Allendoerfer, The Euler number of a Riemannian manifold, Amer. J. Math. 62 (1940), 243-248. MR 2:20e

[AW] C. B. Allendoerfer, A. Weil, The Gauss-Bonnet theorem for Riemannian polyhedra, Trans. Amer. Math. Soc. 53 (1943), 101-129. MR 4:169e

[Be] M. Berger, Riemannian geometry during the second half of the twentieth century, University Lecture Series, 17, American Mathematical Society, Providence, RI, 1998. MR 2000h:53002

[Br] G. E. Bredon, Introduction to compact transformation groups, Pure and Applied Mathematics, Vol. 46, Academic Press, New York-London 1972. MR 54:1265

[C1] S.S. Chern, A simple intrinsic proof of the Gauss-Bonnet formula for closed Riemannian manifolds, Ann. of Math. (2) 45 (1944), 747-752. MR 6:106a

[C2] S. S. Chern, On curvature and characteristic classes of a Riemann manifold, Abh. Math. Sem. Univ. Hamburg 20 (1955), 117-126. MR 17:783e

[F] W. Fenchel, On total curvatures of Riemannian manifolds: I, J. London Math. Soc. 15 (1940), 15-22. MR 2:20f

[G] R. Geroch, Positive sectional curvature does not imply positive Gauss-Bonnet integrand, Proc. Amer. Math. Soc. 54 (1976), 267-270. MR 52:11784

[GS] K. Grove, C. Searle, Positively curved manifolds with maximal symmetry-rank, J. Pure Appl. Algebra 91 (1994), 137-142. MR 95i:53040.

$[\mathrm{H}] \quad$ H. Hopf, Über die Curvatura integra geschlossener Hyperflächen, Math. Ann. 95 (1925), 340-367.

[HS] H. Hopf, H. Samelson, Ein Satz über die Wirkungsräume geschlossener Liescher Gruppen, Comment. Math. Helv. 13 (1941), 240-251. MR 4:3b

[K] S. Kobayashi, Transformation groups in differential geometry, Ergebnisse der Mathematik und ihrer Grenzgebiete, Band 70, Springer, Berlin-Heidelberg-New York 1972. MR 50:8360

[PV] F. Podestà, L. Verdiani, Totally geodesic orbits of isometries, Ann. Global Anal. Geom. 16 (1998), 413-418. MR 99j:53071 
[R] X. Rong, Positively curved manifolds with almost maximal symmetry rank, to appear.

[W] N.R. Wallach, Compact homogeneous Riemannian manifolds with strictly positive sectional curvature, Ann. of Math. 96 (1972), 277-295. MR 46:6243

Fakultät für Mathematik, Ruhr-Universität Bochum, D-44780 Bochum, Germany

E-mail address: puttmann@math.ruhr-uni-bochum.de

Instituto de Matematicas, Unidad Cuernavaca-Unam, Apartado Postal 273-3, Admon. 3, Cuernavaca, Morelos, 62251, Mexico

E-mail address: csearle@matcuer.unam.mx 